\title{
SMART-CAM for Warehouse
}

\author{
Sampada S. Chalke \\ Computer Engineering, \\ $K C$ College of Engineering \\ Management Studies and Research \\ Thane (east) \\ Maharashtra, India - 400603.
}

\author{
Shreyas S. Keny \\ Computer Engineering, \\ $K C$ College of Engineering \\ Management Studies and Research \\ Thane (east) \\ Maharashtra, India - 400603
}

\author{
Sunil R. Patade \\ Zubaida Khan \\ Assistant Professor, \\ Computer Engineering, \\ $K C$ College of Engineering \\ Management Studies and Research \\ Thane (east) \\ Maharashtra, India - 400603
}

\begin{abstract}
We are implementing a project for memory optimization for video surveillance with the help of human motion happening in the surrounding in the warehouse and also will be able to playback the recorded video which will only have the human motion recorded video and images will be click continuously after a fixed interval of time to get a view when there is human motion or no human motion images will be always clicked and stored. This system is based on IoT (Internet of Things). It has a Raspberry Pi (RPI), Camera module to detect human (thief) or detection of fire. Motion detection is a process of confirming a change in the position of an object relative to its surroundings or the change in the surroundings relative to an object. Generally, motion detection is useful in real-time or active surveillance systems. IN this paper the main focus is given to the processing of the captured video data to detect human motion in it. So that it is easy for the user to view images and videos whenever he wants. So this system is very useful to maintain a warehouse and memory optimization will be done.
\end{abstract}

Keywords- motion;memory optimization; warehouse; record.

\section{INTRODUCTION}

Nowadays, maintaining peace in the warehouse and avoiding fire, and avoiding stealing goods in the warehouse is very difficult. It's not possible to properly maintain a warehouse for one man. It's necessary to maintain a warehouse properly and fire-free to avoid a loss. But this system is overcome from this drawback. This system is very helpful, to maintain a proper warehouse and protect it from fire.

This system is based on IoT (Internet of Things). It has a Raspberry Pi (RPI), Camera module to detect human (thief) or detection of fire. Motion detection is a process of confirming a change in the position of an object relative to its surroundings or the change in the surroundings relative to an object. Generally, motion detection is useful in real-time or active surveillance systems. IN this paper the main focus is given to the processing of the captured video data to detect human motion in it.

In this system, the Camera will detect humans only if the movement of humans is happening, otherwise, it cannot detect humans. This system detects human motion with the help of Human detection Sensor. The system only detects if it is human or not. It does not identify the specific person. Haar Cascades is used in this system for person identification. Its technology for the detection of objects related to computer vision. This system records video only if human movement exists. Otherwise, the camera will take a picture of the selected position after the fixed interval of time.
This system also helps in fire detection. If there is smoke or fire in the warehouse, it will detect it and the buzzer of this system gets turned on. It detects fire using a fire sensor that is connected to Raspberry pi. The smoke or fire is detected by the gas sensor. Everything video and pictures clicked by this camera module is stored on the server. So that it is easy for the user to view images and videos whenever he wants. So this system is very useful to maintain a warehouse.

\section{EXISTING SYSTEM}

We undergo the research paper "Video Surveillance Storage: How Much Is Enough?" in 2012 by Seagate shows compression techniques (reduction of actual size by reducing the pixels) which is used to store the CCTV footage which results in loss of quality as there is the reduction of pixels but which does not affect a major the size of the video. So, they suggested using the Backup plus option by Seagate. And other research done in CCTV includes primary and secondary storage systems. Primary storage is connected to the camera from where the data is compressed and stored in a secondary storage which is used for backup which is server or cloud. After some time then the primary is cleaned and indexed to secondary storage. In this technique, data is not lost as CCTV cameras are used for security purposes but the cost of CCTV maintenance increases due to two storages. In recent researches done on this topic, they suggested storing the CCTV footage in the cloud. But this requires a fast internet connection for storing and also costly.

\section{LITERATURE REVIEW}

Real Time Security System Using Human Motion Detection Author ahire upasana1, bagul manisha, gawali mohin November 2015 object tracking is an important component of many vision systems. This system is used not only for visual surveillance but also for augmented reality, traffic control, medical imaging, gesture recognition, etc. after all this the final step of this system is to analyze the content of the video and to identify important events in a scene with real-time detection of motion detection.

A survey on moving object detection and tracking techniques Author A Survey on Moving Object Detection and Tracking Techniques May 2016 Mainly video analysis is categorized into three basic phases: moving entity detecting, finding the trajectory of an object from one frame to another frame, and scrutiny of entity tracks to identify their performance. To Track an object in the static environment (stable) is much easier than tracking objects in a dynamic environment (unstable) i.e. daily public area or more motion area. 
A Survey of Deep Learning-based Object Detection Author Licheng Jiao, Fellow, IEEE, July 2019 Most approaches for moving object detection (MOD) based on computer vision are limited to stationary camera environments. In this advanced driver assistance system (ADAS)the egomotion is added to image frames owing to the use of a moving camera. This results in mixed motion in the image frames and makes it difficult to compare target objects and background.

A Survey of Deep Learning-based Object Detection A Survey of Deep Learning-based Object Detection Oct 2019 This survey focuses on describing and analyzing deep learning-based object detection tasks. The existing surveys always cover a series of the domain of general object detection and may not contain state-of-the-art methods which provide some novel solutions and new directions of this task.

\section{PROPOSED SYSTEM}

Our system focused on memory optimization with the help of motion and in motion, we focused on the human motion especially when there is human motion our system stores the footage and when there is no human motion there is no storage of footage occurs due to which memory is optimized and onside a continuous images is clicking occurs after a fixed interval of time so following are processes and hardware execution.

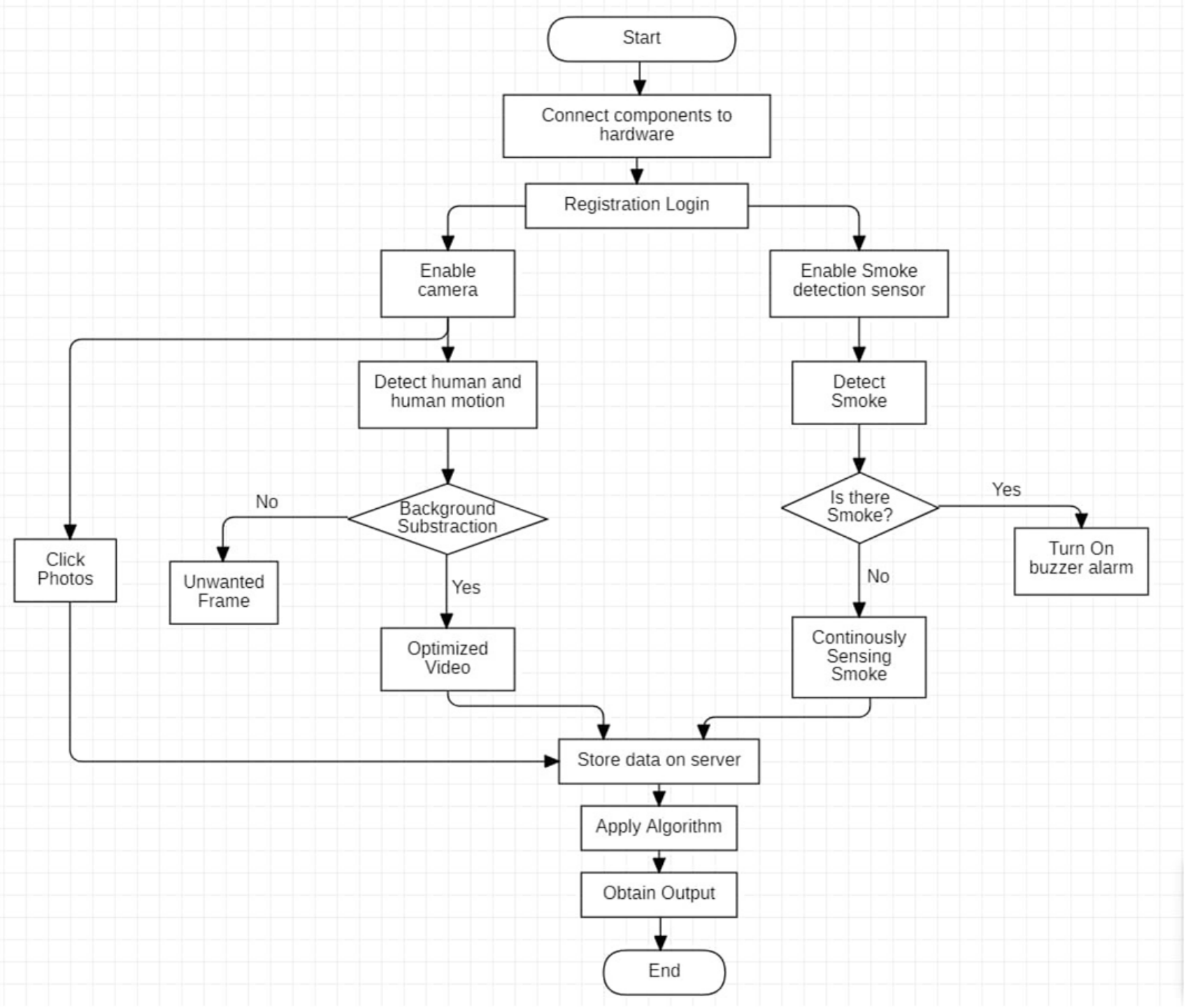

Fig. 1. Proposed System Flowchart

\section{A. Raspberry PI 3 MODEL B+}

In this the Smart-Cam surveillance system Raspberry Pi 3 is the major working component which is the heart of the system which can be work like the minicomputer, also there are a few more model but in this model, Raspberry Pi 3 Model $\mathrm{B}+$ has some important hardware features which are
Ethernet port, 4 USB 2.0 ports, HDMI output, audio input and output, and general-purpose input and output (GPIO) and pins along the top edge of the board. These pins are an interface between the $\mathrm{Pi}$ and the outside environment and we can turn on or off i.e. input or Pi can turn on or off i.e. output, the 40 pins, 26 are GPIO pins, and the others are power or ground pins plus two ID EEPROM pins. 


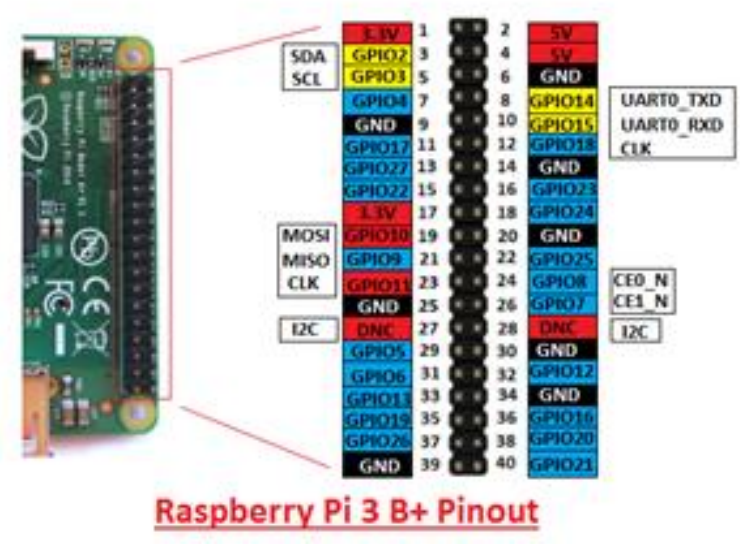

Fig. 2. Raspberry Pi 3B+ Pinout

\section{B. Fire Detection and Giving Buzzers Alert}

The warehouse is the one place where fire accidents could happen so for detection of fire we used this sensor DHT11 is the sensor we used to measure the temperature and humidity. This sensor has a resistive humidity sensing component and a negative temperature coefficient (NTC) and there is an 8 bit MCU that is connected to responsible for the fast response. It is a lowcost device but gives an effective value for both temperature and humidity at a time. This sensor aims to detect fire or fire flame if occurs in the warehouse. Once the fire detection sensor gets active and gives the output true (i.e. detects the fire flame) then the buzzer will get active and will give a loud alert.

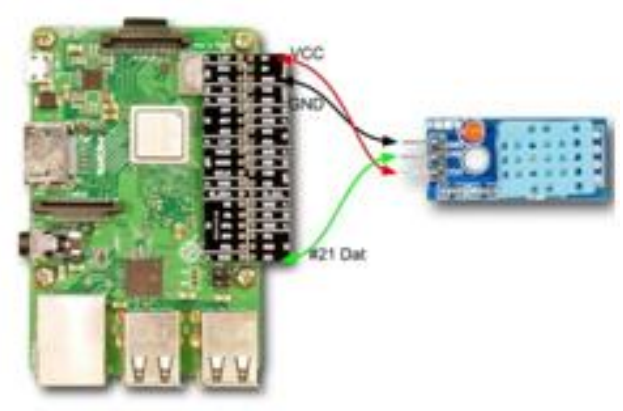

Fig. 3.

\section{Human Motion Detection Using PIR Sensor}

Our system main focused is human motion detection so to get an accurate human detection motion video we used a PIR sensor to detect a human motion detection around. This sensor is made of a pyroelectric sensor, which is used to detect infrared radiation. PIR sensors are helpful and effective and they are flat control and minimal effort, have a wide lens range and are simple to interface with. When there is the presence of human IR radiations around the sensor, the sensor detects the human radiations and then converts it directly to electrical pulses and fed to the inverter circuit. This inverter circuit consists of a transistor to gets into saturation with application of high base current and eventually develops a low collector voltage so the transistor output is low. This low inverter output is connected to the model. Based on the input received by the model, video is fetched from the camera.

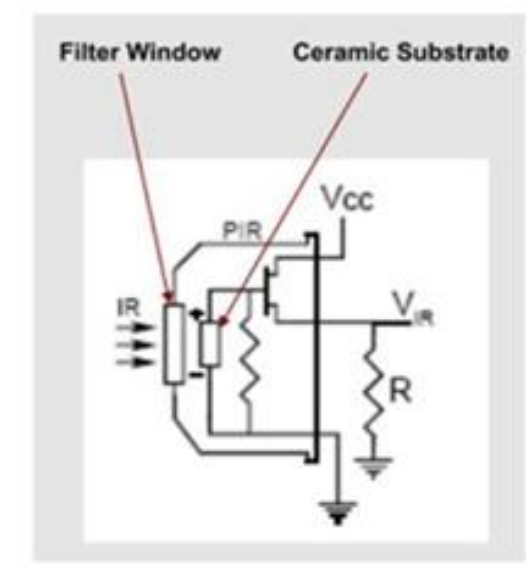

Fig. 4.

\section{METHODOLOGY}

\section{A. Memory Optimization Using Background Subtraction}

Our system memory optimization is dependent on the background subtraction algorithm is one of the most important parts of our image and video processing. Some unnecessary parts during the image or video processing should be removed because they more required memory which is not useful to use. This algorithm undergoes from following stages video is first taken as input the video is the human motion video which is obtained with the help of PIR sensor in next stage conversion frames occur in this frames are separated into frames current frame and background frames and after that background separation stage occurs in this stage if the current frame is same with background frame then it is discarded and if the current frame is not same with background frame then it is saved in storage and final stage optimize video is obtained and saved.

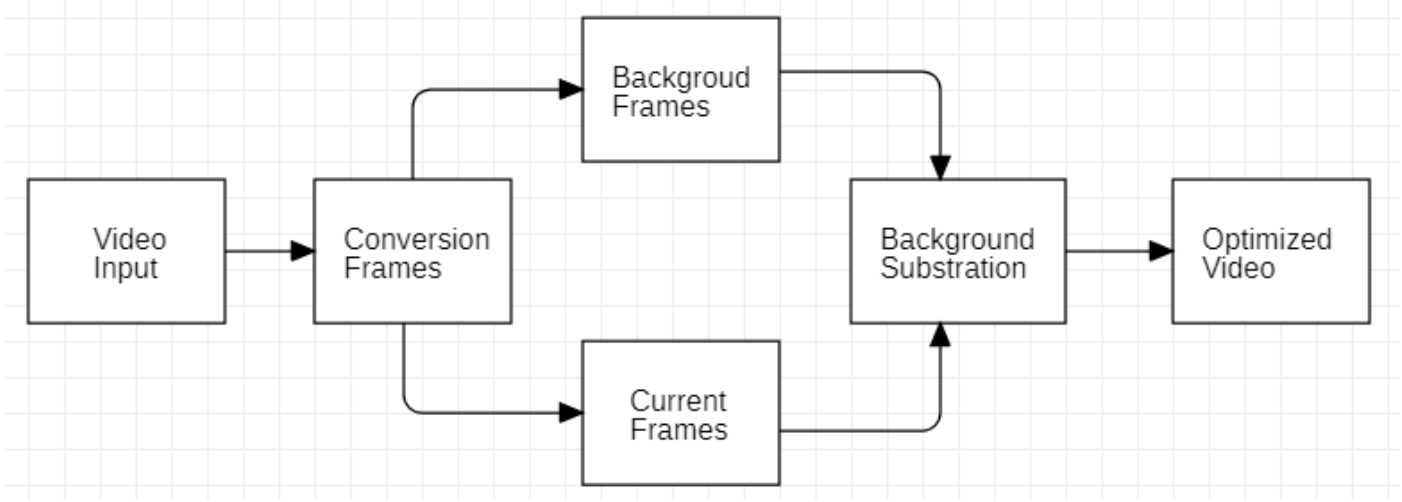

Fig. 5. 


\section{B. Algorithm}

Step 1: Start.

Step 2: Input video - video is fetched.

Step 3: Frame Separation - In this step video is converted into continuous frames

Step 4: Image Sequence - after frame separation images are sequence into to part a current frame which is changes according to the time and one background frame which is the mainframe which is to check the difference and used to calculate the difference.

Step 5: background subtraction - based on the difference of frame between the current frame and background frame optimization occur that is if the background frame and current frame are the same then the current frame is discarded and if the background and current frame is a difference then the frame is stored hence due to this condition memory is optimized.

Step 6: optimized video - Finally the optimized video is obtained and stored in the storage.

Step 7: end

\section{CONCLUSION}

Thus, we've got developed a sensible surveillance camera that finds humans and their motion we additionally use smoke detection that is activated buzzer once fire/smoke detector detects fire/smoke. Also, the camera system is compact and can be implemented at a low cost. The implemented human detection rule is extremely effective, with high accuracy which may be enhanced more by effectively improving the illumination of the area.

\section{REFERENCES}

[1] Seshapu Prasad, D.Suneel, "Motion Sensor Based Security Lock And Theft Detection". International Journal Of Science Technology And Management.Vol No.4, Issue No.01

[2] Abhilasha A Sayar ,Dr. Sunil N Pawar , “ Review Of Bank Locker System Using Embedded System' 'International Journal Of Advanced Research In Computer And Communication Engineering

[3] Seshapu Prasad, D.Suneel, "Motion Sensor Based Security Lock And Theft Detection". International Journal of Science Technology and Management.Vol No.4, Issue No.01

[4] Abhilasha A Sayar ,Dr. Sunil N Pawar, " Review Of Bank Locker System Using Embedded System', International Journal Of Advanced Research In Computer And Communication Engineering

[5] Zorzi, Ai Emulates The Human Brain,Https://Erc.Europa.Eu/Projectsand -Results/Erc-Stories/SelfLearning-Ai Emulates-Human-Brain Accessed: February 24, 2017.

[6] Robotics, Vol.2 Issue 2, pp: 112-117, 2014.

[7] R. Szeliski, Computer Vision: Algorithms and Applications Http://Szeliski.Org/Book/Accessed: February 18, 2017.

[8] Victor, E. (2012), Real-time Computer Vision with OpenCV, Volume 10 Issue 4, Accessed: April 16, 2017

[9] Abhilasha A Sayar, Dr. Sunil N Pawar "Review Of Bank Locker System Using Embedded System' 'International Journal Of Advanced Research In Computer And Communication Engineering 